\title{
Population Structure of Fusarium graminearum from Maize and Rice in 2009 in Korea
}

\author{
Seung-Ho Lee ${ }^{1}$, Jungkwan Lee ${ }^{2}$, Young Ju Nam ${ }^{1}$, Soohyung Lee ${ }^{1}$, Jae-Gee Ryu ${ }^{1}$ and Theresa Lee ${ }^{1 *}$ \\ ${ }^{1}$ Microbial Safety Division, National Academy of Agricultural Science, RDA, Suwon 441-707, Korea \\ ${ }^{2}$ Center for Fungal Pathogenesis, Seoul National University, Seoul 151-921, Korea \\ (Received on November 12, 2010; Accepted on November 23, 2010)
}

We performed diagnostic PCR assays and a phylogenetic analysis using partial sequences of TEF1 (translation elongation factor-1) to determine the trichothecene chemotypes and genetic diversity of $\boldsymbol{F}$. graminearum isolates from maize and rice samples collected in 2009 in Korea. PCR using a species-specific primer set revealed a total of 324 isolates belonging to the putative $F$. graminearum species complex. PCR with trichothecene chemotypespecific primers revealed that the nivalenol (NIV) chemotype was predominant among the fungal isolates from rice $(95 \%)$ in all provinces examined. In contrast, the predominant chemotype among the corn isolates varied according to region. The deoxynivalenol (DON) chemotype was found more frequently $(66 \%)$ than the NIV chemotype in Gangwon Province, whereas the NIV chemotype $\mathbf{( 7 0 \% )}$ ) was predominant in Chungbuk Province. Phylogenetic analysis showed that all DON isolates examined were clustered into lineage 7 , while the NIV isolates resided within lineage 6 ( $F$. asiaticum). Compared with previous studies, the lineage 6 isolates in rice have been predominantly maintained in southern provinces, while the dominance of lineage 7 in maize has been evident in Gangwon at a slightly reduced level.

Keywords : Fusarium asiaticum, F. graminearum, lineage, maize, rice, $T E F 1$

Fusarium graminearum (telomorph Gibberella zeae) causes Fusarium head blight of barley (FHB), wheat and rice and ear rot of maize (Leslie and Summerell, 2006). In addition to reduced grain yield and quality, infected grains are highly likely contaminated with mycotoxins. F graminearum species often exist in a complex, producing trichothecene mycotoxins such as deoxynivalenol (DON), nivalenol (NIV), and their acetylated derivatives (Bottalico, 1998).

The major species most associated with FHB comprise the $F$. graminearum complex, which is subdivided into at least twelve phylogenetically distinct species (O'Donnell et

\footnotetext{
*Corresponding author.

Phone)+82-31-290-0451, FAX) +82-31-290-0407

E-mail) tessyl1@korea.kr
}

al., 2008). Other F. graminearum complexes may be associated with FHB, but only a few species are known to be pathogenic (Bottalico, 1998). Studies of the population structure of $F$. graminearum have been performed intensively since 2000 and have focused mainly on lineage characterization and mycotoxin chemotypes (Starkey et al., 2007). F. graminearum (lineage 7) is the dominant species associated with FHB in the US, Europe, and Brazil, whereas $F$. asiaticum (lineage 6) is the primary species in Asia (Karugia et al., 2009; O'Donnell et al., 2000, 2004; Scoz et al., 2009; Ueda et al., 2007; Ward et al., 2008; Yang et al., 2008; Yli-Mattila et al., 2009). In Korea, the lineage composition of $F$. graminearum populations from maize and rice have been studied (Lee et al., 2004; Lee et al., 2009).

Because change in fungal population structure affects disease development, regular monitoring of pathogen populations is essential for disease management. Due to global climate change, which favors fungal growth, many crops, including cereals face an unusual cultivation environment and more fungal infections. Recent and frequent outbreaks of FHB in cereals in Korea could be a consequence. Therefore, the purposes of this work were (1) to investigate the trichothecene chemotypes of $F$. graminearum from maize and rice sampled in 2009, (2) analyze the lineages of the isolates and genetic diversity of the populations, and (3) determine the geographical distribution of dominant $F$. graminearum populations in Korean maize and rice.

\section{Materials and Methods}

Plant sampling. Grain samples (10 $\mathrm{g}$ for rice, $30 \mathrm{~g}$ for maize) were collected from 32 rice fields in eight provinces (Gangwon, 4; Gyeonggi, 8; Chungbuk, 2; Chungnam, 4; Gyeongbuk, 2; Gyeongnam, 3; Jeonbuk, 7; Jeonnam, 2) and from 19 maize fields in eastern (Gangwon, 15) and midland (Chungbuk, 4) provinces of Korea in 2009. All samples were dried and stored at $4{ }^{\circ} \mathrm{C}$ until isolation of the fungi.

Isolation of fungi. A total of 105 seeds from each sample 
were soaked in $1 \%$ sodium hypochlorite for $2 \mathrm{~min}$, rinsed twice in sterile water for $2 \mathrm{~min}$ each, placed on potato dextrose agar (PDA; Difco Laboratories, Detroit, MI) plates containing streptomycin $(600 \mu \mathrm{g} / \mathrm{ml})$ and incubated at $25^{\circ} \mathrm{C}$. After five days, Fusarium candidates were isolated by transferring the mycelia from PDA or carnation leaf agar. After five to seven days of growth at $25^{\circ} \mathrm{C}$, a spore suspension was prepared in sterilized water and spread on water agar for single spore isolation. To obtain monosporic culture, a single germinated conidium was transferred to PDA. The fungal isolation was maintained on PDA at $4^{\circ} \mathrm{C}$ and stored as frozen conidial suspensions in $20 \%$ glycerol at $-80^{\circ} \mathrm{C}$.

PCR assays. Fungal DNA was isolated from a three-day mycelial culture in complete medium (CM) (Leslie and Summerell, 2006) broth using the CTAB method (Proctor et al., 1995). The concentration of each DNA sample was adjusted to $20 \mathrm{ng} / \mu \mathrm{l}$ for use in PCR. The primers used in this study were synthesized by the Bioneer oligonucleotide synthesis facility (Daejeon, Korea). A PCR assay with a $F$. graminearum-specific primer pair ( Fg16) was performed as described by Nicholson et al. (1998). To identify the trichothecene chemotype, PCR was performed using primer pairs derived from TRI13 and TRI7 which are essential for trichothecene biosynthesis as described previously (Kim et al., 2003; Lee et al., 2001; Lee et al., 2002). Amplification of the partial sequence of the gene encoding elongation factor 1-alpha (TEFl) was performed with the primers TEF-F (5'-ATGGGTAAGGARGACAAGAC-3') and TEFR (5'-GGARGTACCAGTSATCATGTT-3') using the method described by O'Donnell et al. (1998). Each TEF1 PCR product was purified using a QIAquick ${ }^{\circledR}$ PCR Purification Kit (QIAGEN, Valencia, CA) according to the manufacturer's protocol, and its nucleotide sequence determined (Macrogen Inc., Seoul, Korea). The TEF1 sequences obtained from the fungal isolates were aligned with those from $F$. graminearum species complex isolates archived in the FUSARIUM-ID database (http://isolate.fusariumdb.org/ index.php; Geiser et al., 2004) using CLUSTALW (Thompson et al., 1994).

Phylogenetic analyses. Partial nucletotide sequences of TEF1 (426 bp) were subjected to a phylogenetic analysis using unweighted pair grouping by mathematical average (UPGMA) in the MEGA4 program (Nei and Kumar 2000; Tamura et al., 2007). Bootstrap analyses were performed with 1,000 replicates to assess the support for subgroups. The data set included TEF1 sequences from the reference strains of each chemotype and lineage. Both Korean strains 88-1 and SCK04 [belonging to the F. graminearum complex lineage 6 (F. asiaticum)] were of the NIV chemotype, while the US strains GZ3639 and PH-1 (F. graminearum lineage 7) were of the DON chemotype. In addition, previously published strains were included: lineage 2 (F. meridionale 29010 and 28721), lineage 3 (F. boothii 29011 and 29105), lineage 6 (F. asiaticum 13818 and 28720), and lineage 7 ( $F$. graminearum 5883 and 28439) (O'Donnell et al., 2000).

\section{Results}

Isolation of Fusarium species from maize and rice. Among the Fusarium isolates collected from 19 maize and 32 rice fields, a total of 324 isolates (266 from rice and 58 from maize) were identified as $F$. graminearum based on a species-specific diagnostic PCR assay (Table 1). These isolates were used all for trichothecene chemotyping and a subset of the isolates was used for further study. Other Fusarium species were isolated as well, but they were not identified at the species level.

Trichothecene chemotyping of $\boldsymbol{F}$. graminearum isolates. PCR with TRI13- and TRI7-specific primers revealed that $47 \%$ ( 27 of 58 ) of the maize isolates examined and $95 \%$ ( 253 of 266) of the rice isolates were NIV chemotype (Table 1). In rice, the NIV chemotype was predominant in all provinces tested. However, the DON chemotype was found in the middle and eastern provinces only. In maize, the ratio of DON to NIV chemotypes varied by region; the DON chemotype $(66 \%)$ was predominant in Gangwon Province, while the NIV chemotype (70\%) was predominant in Chungbuk Province (Table 1).

Phylogenetic analysis and lineage composition. From the 324 putative $F$. graminearum isolates identified by diagnostic PCR, we analyzed the population structure of 142 (48

Table 1. Distribution of F. graminearum chemotypes based on TRI13 in maize and rice

\begin{tabular}{lcccc}
\hline \multirow{2}{*}{ Province } & \multicolumn{2}{c}{ Rice } & \multicolumn{2}{c}{ Maize } \\
\cline { 2 - 5 } & DON & NIV & DON & NIV \\
\hline Gangwon & 2 & 8 & 25 & 13 \\
Gyeonggi & 4 & 60 & $-^{\mathrm{a}}$ & - \\
Chungbuk & 1 & 10 & 6 & 14 \\
Chungnam & 0 & 62 & - & - \\
Gyeongbuk & 5 & 14 & - & - \\
Gyeongnam & 0 & 12 & - & - \\
Jeonbuk & 1 & 74 & - & - \\
Jeonnam & 0 & 13 & - & - \\
\hline Total & 13 & 253 & 31 & 27 \\
\hline
\end{tabular}

${ }^{\mathrm{a}}$ not sampled 
Table 2. Lineage and trichothecene chemotype composition of the $F$. graminearum populations from maize and rice

\begin{tabular}{ccccc}
\hline \hline \multirow{2}{*}{ Lineage } & $\begin{array}{c}\text { No. of } \\
\text { isolates }\end{array}$ & TRI7 & TRI13 \\
\hline \multirow{6}{*}{ F. incarnatum } & 1 & NIV & NIV \\
& & 2 & NIV & NIV \\
& Fusarium sp. & 1 & na* & NIV \\
& Total & 48 & na & NIV \\
\cline { 2 - 5 } Rice & 6 & 78 & NIV & NIV \\
& & 1 & NIV & DON \\
& & 1 & DON & DON \\
& 7 & 7 & DON & DON \\
& & 1 & NIV & NIV \\
& & 3 & na & NIV \\
& F. incarnatum & 1 & na & na \\
& & 1 & na & na \\
& F. negundis & 1 & na & na \\
\cline { 2 - 5 } Fusarium sp. & Total & 94 & & \\
\cline { 2 - 5 } & & & &
\end{tabular}

*na, not amplified.

from maize and 94 from rice) based on TEF1 gene sequences (Table 2). The isolates were selected as multiple clonal representatives of each region collected, cultivation method, or host cultivar. Among the sequences analyzed, those of 61 isolates (clonal single representatives; 20 from maize and 41 from rice) were used to draw phylograms in Figs. 1-4.

Phylogenetic trees generated using the TEF1 sequences (426 bp) of the isolates by the UPGMA method displayed a topology consisting of several distinctive clades, each corresponding to the lineages of the previously identified $F$. graminearum species complex (Figs. 1 and 2); the lineage 3 reference strains were not separated from the lineage 7 isolates in maize tree. Most isolates from both plant hosts were separated into two major lineages (lineage 7 and lineage 6) (Figs. 1 and 2). This result is consistent with the pattern of trichothecene chemotype (Table 2). However, no isolates examined were separated by geographical origin.

Among the maize isolates, the largest group was lineage 7 (L7) with 29 isolates, followed by lineage 6 (L6) with 15 isolates, corresponding to a 2:1 ratio between the two lineages (Table 2). Most of the L7 and L6 isolates were of the DON and NIV chemotypes, respectively. Neither L2 nor L3 isolates were found in the maize samples. Remarkably, one NIV-chemotype isolate (SM9) was not clustered within any lineage subclade identified in the tree. In addition, other Fusarium species (three $F$. incarnatum isolates) were reidentified from the putative $F$. graminearum collection in maize (Table 2).

In contrast, the rice isolate populations consisted mainly of the L6 group with 80 isolates, and only eight isolates were grouped in L7, generating a 1:10 ratio between L7 and

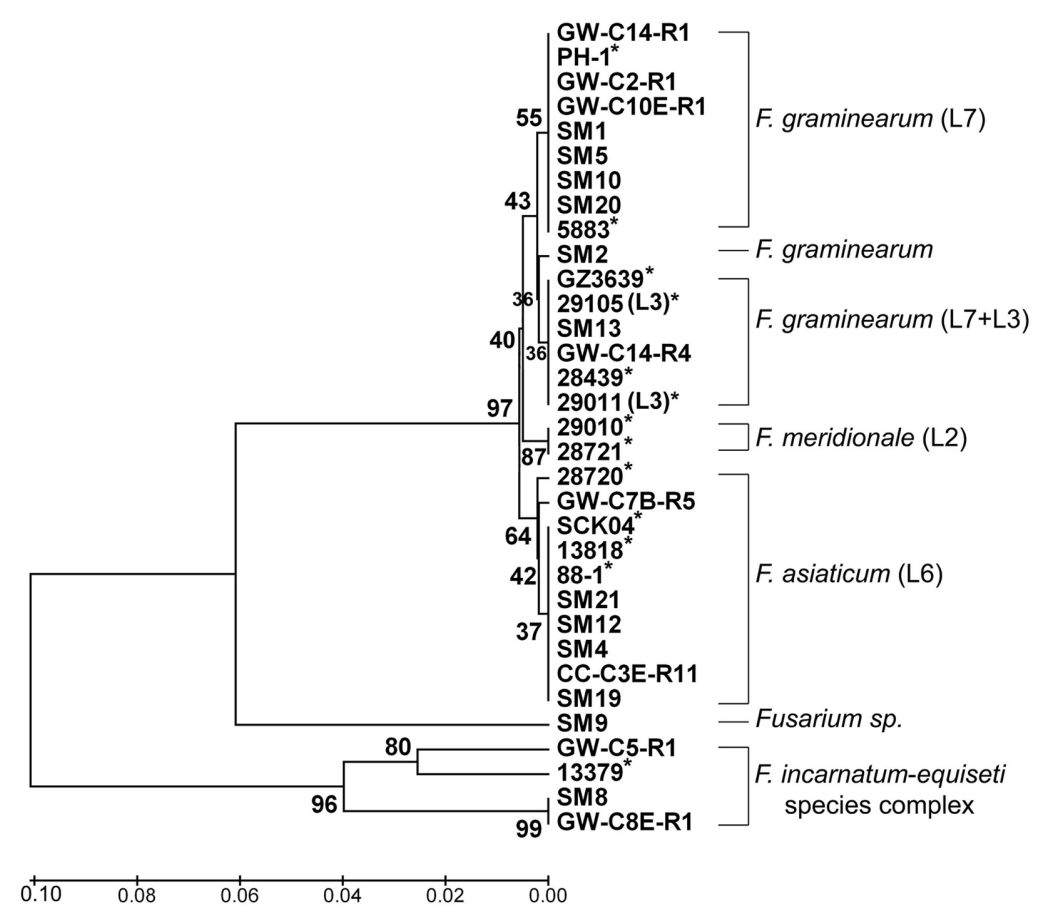

Fig. 1. UPGMA tree based on the TEF1 sequences from maize $F$. graminearum isolates. Bootstrap frequencies are shown above the branches. The scale bar shows the genetic distance between the isolates. The transition weight was set at 1 . Reference isolates with known lineages are marked with asterisk $(*)$. 


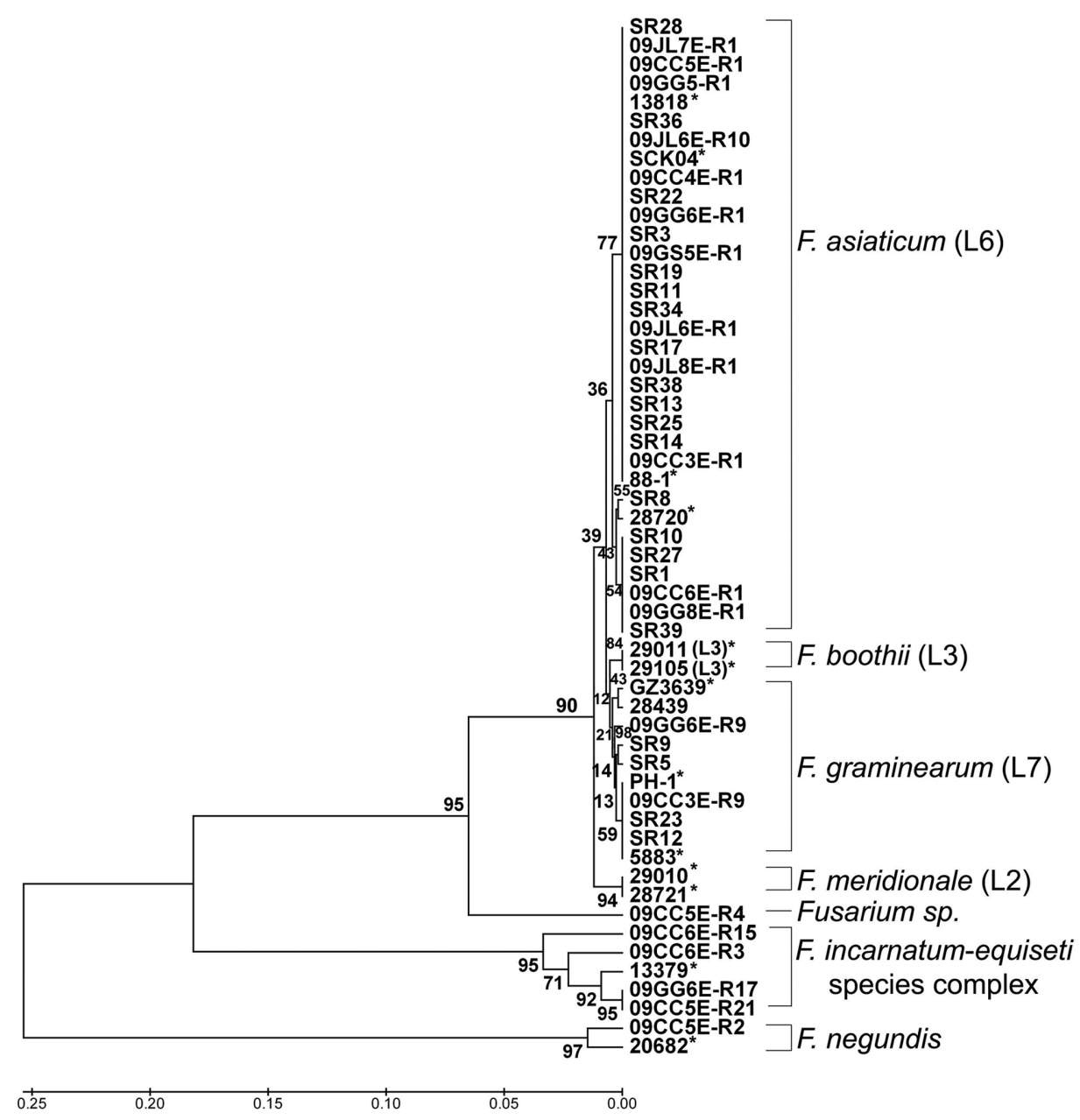

Fig. 2. UPGMA tree based on the $T E F 1$ sequences from rice $F$. graminearum isolates. Bootstrap frequencies are shown above the branches. The scale bar shows genetic distance between the isolates. Transition weight was set at 1 . The reference isolates with known lineages are marked with asterisk $(*)$.

L6. Interestingly, there was a subclade in the lineage 6 group, which consisted solely of Korean isolates. Three isolates showed an inconsistency between lineage and chemotype: two DON chemotype isolates of L6 (09JL6ER10 and SR8), and an NIV chemotype isolate of L7 (SR12) (Table 2). As in the case of the maize isolates, no lineage 2 or 3 isolates were found. Two other Fusarium species (four $F$. incarnatum and one $F$. negundis isolates) were reidentified from the putative $F$. graminearum collection in rice.

Genetic diversity within lineage. To determine the level of genetic diversity within each lineage, isolates in the same lineage from both hosts were analyzed by UPGMA clustering (Figs. 3 and 4). Within L7, 60\% of the Korean isolates (10 of 17) were clustered into one subclade with $54 \%$ bootstrap support (BS). However, rest of the isolates were clustered separately and not supported strongly (Fig. 3).
The first subclade consisted of isolates from maize and rice, implying little host effect on the genetic diversity of the L7 population. An NIV chemotype isolate (SR12) was also clustered into L7. Two DON chemotype isolates from lineage 6 (09JL6E-R10 and SR8) was included to see relation with lingeage 7 and they were clustered into L6.

In contrast, the phylogenetic tree of the L6 isolates showed that $73 \%$ of the Korean isolates ( 27 of 37 ) were clustered into the largest clade with $41 \%$ BS (Fig. 4). The smaller clade consisted of isolates only from rice. Intentional inclusion of one NIV chemotype isolate (SR12, lineage 7) was clustered into L7.

\section{Discussion}

Phylogenetic analyses of the $F$. graminearum populations from maize and rice enabled a comparison of maize with rice in terms of lineage composition and genetic diversity. 


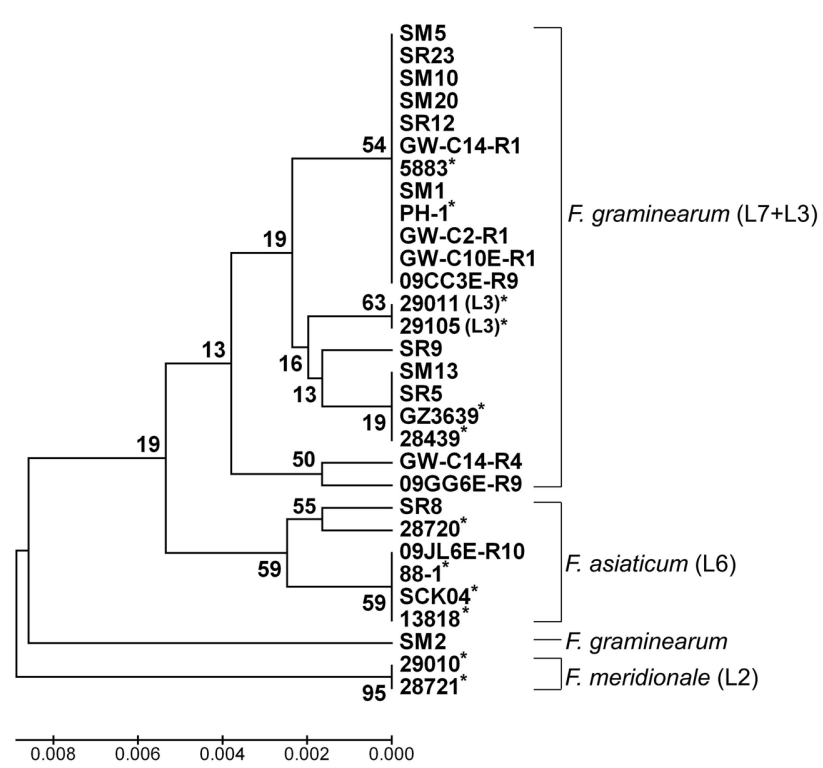

Fig. 3. UPGMA tree generated with $F$. graminearum (L7) and additional DON chemotype isolates. Bootstrap frequencies are shown above the branches. The scale bar shows genetic distance between the isolates. Transition weight was set at 1. The reference isolates with known lineages are marked with asterisk (*). Two DON type lineage 6 isolates (SR8 and 09JL6E-R10) were included for comparison.

As expected from previous reports, the dominant populations in maize were lineage 7, while the lineage 6 or $F$. asiaticum isolates were predominant in rice. The prevalence of lineage 6 populations in rice and barley, and lineage 7 in maize has been observed in Korea (Lee et al., 2004; Lee et al., 2009). The predominance of lineage 6 in cereals other than maize has also been observed, especially in Asia, including in China and Japan. In eastern China, all fungal isolates from diseased wheat heads belonged to lineage 6 (Gale et al., 2002). A survey of a large number of isolates throughout China revealed the unequal distribution of Fusarium lineages 6 and 7 in wheat (Qu et al., 2008), and $F$. asiaticum populations showed an association between mycotoxin chemotype and geographic location in barley in China (Yang et al., 2008). In Japan, isolates from wheat and barley were mostly lineage 6 and approximately $70 \%$ of the populations were of the NIV chemotype (Ueda et al., 2007; Suga et al., 2008).

A comparison of our data with those from previous studies suggests that lineage composition within the maize population has been changed during the last ten years in Korea. A previous study in Gangwon Province, an eastern area where maize is a major crop, reported that lineage 7 outnumbered lineage 6 by more than six times from 1999 to 2001 (a result generated using about 500 isolates; Lee et al., 2004). The ratio of $6: 1(430: 72)$ between lineages 7 and 6

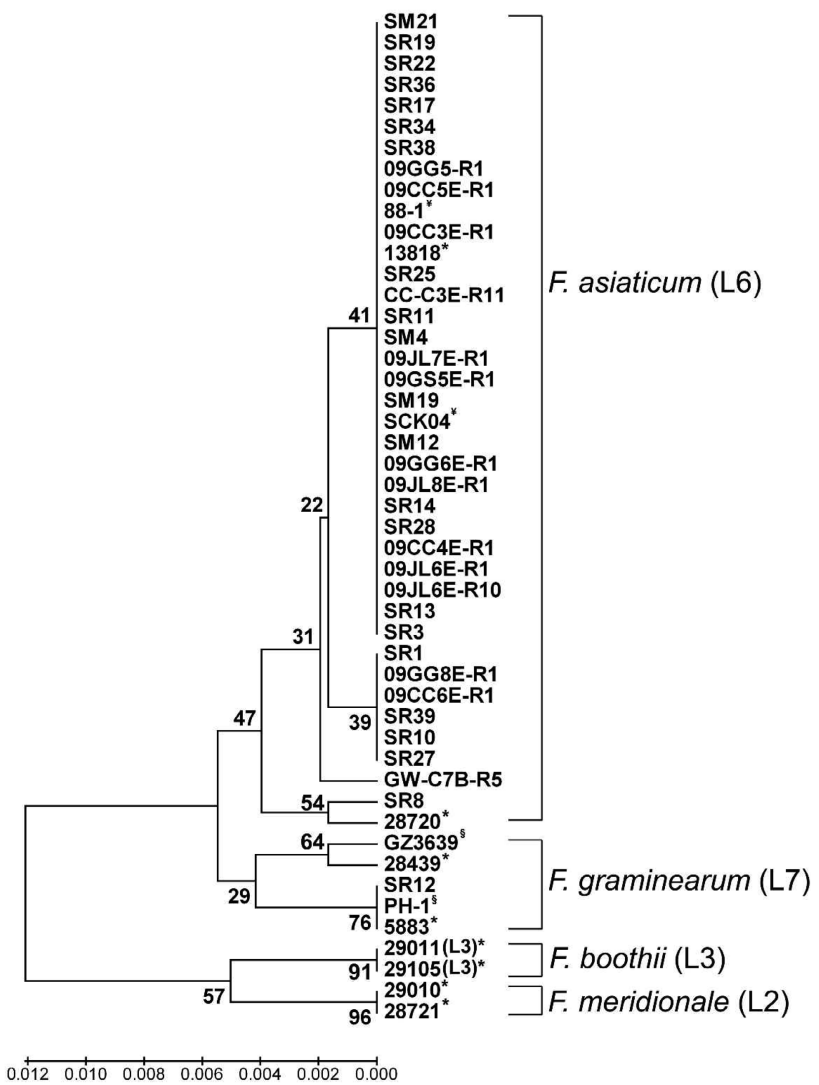

Fig. 4. UPGMA tree generated with $F$. asiaticum (L6) and additional NIV chemotype isolates. Bootstrap frequencies are shown above the branches. The scale bar shows genetic distance between the isolates. Transition weight was set at 1 . The reference isolates with known lineages are lineages are marked with asterisk $(*)$. An NIV type lineage 7 isolate (SR12) was included for comparison.

was changed to 5:1 (23:5) in this study, indicating a slight decrease in the population size of lineage 7 and an increase in lineage 6 in Gangwon Province. However, the sample size examined in this study was relatively small, thus it is necessary to analyze more isolates for confirmation of the lineage composition. The fungal population structure in Chungbuk Province, which is located in central Korea immediately below Gangwon Province, differed from Gangwon in that the ratio of lineage 7 to 6 was $3: 5$ (6:10) (data not shown). The difference between the populations from Gangwon and Chungbuk may reflect major crop difference between these provinces. In Chungbuk, rice, as opposed to maize, is a major crop, although this province is another maize-cultivating area in Korea. The difference in lineage composition is more evident when compared with that in provinces where very little maize has been cultivated. This study also supports the previous result that lineage composition of the F. graminearum populations is host- and location-dependent in Korea (Lee et al., 2009). 
Most isolates from southern rice fields were of lineage 6, ranging from 99 to $100 \%$ as described previously (Lee et al., 2004; Lee et al., 2009), demonstrating no practical change in the dominant rice isolate population during the last ten years. In 2001, lineage 6 was found at a relatively lower frequency $(80 \%)$ in rice samples from Gangwon (Lee et al., 2009). In 2009, five of seven Gangwon isolates were lineage 6, showing similar frequency as in 2001.

The fungal populations from the maize samples in this study consisted of only two lineages, which is comparable with data from a study (done from 1999 till 2001) showing almost equal numbers of lineage 3 and a few lineage 2 isolates (Lee et al., 2004). However, the lack of diverse lineages in the 2009 samples could be attributed to the smaller sampling size. Another possible reason may be due to difference on approaches used in the studies. We used TEF1 gene analysis to differentiate the lineage of each isolate while the previous study performed AFLP. Even though AFLP method is one of the best approaches to get comprehensive understanding about genetic diversity of fungal populations, it is time consuming and laborious. The analysis of TEF1 gene sequences in this study was able to provide the identification of lineage 6 isolates from other $F$. graminearum lineages, and it can be simply used for population studies as integrating other structural genes.

A phylogenetic analysis within lineage 6 or 7 , however, did not reveal a level of genetic diversity among the lineage 6 isolates or among the lineage 7 isolates. The subclades within lineage 6 were poorly supported with BS values (less than 50\%) and BS values for ones within lineage 7 were similar. Such poor clustering within lineage 6 may suggest that the lineage 6 isolates are not genetically diverse. In a study by Lee et al. (2009) using AFLP analysis, high genetic identity among populations and low genotype diversity was observed among Korean lineage 6 rice isolates. Regardless of the level of genetic diversity within lineage 6 , this result implies that dominant lineage 6 isolates have been evolving with rice, possibly aided by outcrossing, adaptation in rice, or overwintering. Clustering of lineage 6 having DON chemotype with lineage 6 clade rather than DON type and of lineage 7 having NIV chemotype with lineage 7 is consistent with that trichothecene chemotype is not lineage dependent (Lee et al., 2009; Ward et al., 2002).

This report is the first phylogenetic analysis of $F$. graminearum populations in Korea to use the nucleotide sequence of a DNA marker (TEF1). However, the relatively low BS values for each lineage clade in the phylogenetic trees in this study suggest that additional analyses are necessary. In particular, the lack of a clear separation between the L3 and L7 clades in the trees suggests that either TEF1 sequence (426 bp) is not long enough for phylo- genetic analysis, or that UPGMA is not the best algorithm for this purpose, although it generated more reliable trees compared with those generated by Neighbor-joining or Maximum-parsimony methods. Additional DNA markers with longer sequences will be useful to further dissect the Korean populations. In China, F. asiaticum from barley was analyzed with VNTR markers, revealing high genetic diversity and substructures (Zhang et al., 2010).

Overall, because rice has been cultivated throughout Korea and lineage 6 is known to have better fitness in rice than in maize, rice should provide a better niche for lineage 6 (F. asiaticum). This relation between lineage 6 and rice may be contributing to the evolution of the population, which favors the growth of endemic $F$. asiaticum with better fertility. Therefore, further study to dissect $F$. asiaticum populations at more complicated levels is needed with a long-range plan in Korea.

\section{Acknowledgments}

This work was supported by research grants (PJ006897 and PJ007340) from the Rural Development Administration of the Republic of Korea.

\section{References}

Bottalico, A. 1998. Fusarium diseases of cereals: Species complex and related mycotoxin profiles, in Europe. J. Plant Pathol. 80:85-103.

Gale, R. L., Chen, L. F., Hernick, C. A., Takamura, K. and Kistler, H. C. 2002. Population analysis of Fusarium graminearum from wheat fields in eastern China. Phytopathology 92:13151322.

Geiser, D. M., Jiménez-Gasco1, M. M., Kang, S., Makalowska, I., Veeraraghavan, N., Ward, T. J., Zhang, N., Kuldau, G. A. and O’Donnell, K. 2004. FUSARIUM-ID v. 1.0: A DNA sequence database for identifying Fusarium. Eur. J. of Plant Pathol. 110:473-479.

Karugia, G. W., Suga, H., Gzle, L. R., Nakajima, T., Ueda, A. and Hyakumachi, M. 2009. Population structure of Fusarium asiaticum from two Japanese regions and eastern China. J. Gen. Plant Pathol. 75:110-118.

Kim, H. S., Lee, T., Dawlatana, M., Yun, S. H. and Lee, Y. W. 2003. Polymorphism of trichothecene biosynthesis genes in deoxynivalenol- and nivalenol-producing Fusarium graminearum isolates. Mycol. Res. 107:190-197.

Lee, J., Chang, I. Y., Kim, H., Yun, S. H., Leslie, J. F. and Lee, Y. W. 2009. Genetic diversity and fitness of Fusarium graminearum populations from rice in Korea. Appl. Environ. Microbiol. 75:3289-3295.

Lee, T., Han, Y. K., Kim, K. H., Yun, S. H. and Lee, Y. W. 2002. Tri13 and Tri7 determine deoxynivalenol and nivalenol producing chemotypes of Gibberella zeae. Appl. Environ. Microbiol. 68:2148-2154. 
Lee, T., Oh, D. W., Kim, H. S., Lee, J., Kim, Y. H., Yun, S. H. and Lee, Y. W. 2001. Identification of deoxynivalenol and nivalenol producing chemotypes of Gibberella zeae using PCR. Appl. Environ. Microbiol. 67:2966-2972.

Lee, Y. W., Jeon, J. J., Kim, H., Jang, I. Y., Kim, H. S., Yun, S. H. and Kim, J. G. 2004. Lineage composition and trichothecenes production of Gibberella zeae population in Korea, p.117-122. In T. Yoshizawa (ed.), New horizons of mycotoxicology for assuring food safety. Japanese Association of Mycotoxicology, Kagawa, Japan.

Leslie, J. F. and Summerell, B. A. 2006. The Fusarium laboratory manual. Blackwell Publishing, Ames, IA, USA.

Nei, M. and Kumar, S. 2000. Molecular Evolution and Phylogenetics. Oxford University Press, New York.

Nicholson, P., Simpson, D. R., Weston, G., Rezanoor, H., Lees, N., Parry, A. K. and Joyce, D. W. 1998. Detection and quantification of Fusarium culmorum and Fusarium graminearum in cereals using PCR assays. Physiol. Mol. Plant Pathol. 53:17-37.

O’Donnell, K., Kistler, H. C., Cigelnik, E. and Ploetz, R. C. 1998. Multiple evolutionary origins of the fungus causing Panama disease of banana: Condordant evidence from nuclear and mitochondrial gene genealogies. Appl. Biol. Sci. 95:20442049.

O’Donnell, K., Kistler, H. C., Tacke, B. K. and Casper, H. H. 2000. Gene genealogies reveal global phylogeographic structure and reproductive isolation among lineages of Fusarium graminearum, the fungus causing wheat scab. Proc. Natl. Acad. Sci. USA 97:7905-7910.

O’Donnell, K., Ward, T. J., Geiser, D. M., Kistler, H. C. and Aoki, T. 2004. Genealogical concordance between the mating-type locus and seven other nuclear gene supports formal recognition of nine phylogenetically distinct species within the Fusarium graminearum clade. Fungal Genet. Biol. 41:600-623.

O’Donnell, K., Ward, T. J., Aberra, D., Kistler, H. C., Aoki, T., Orwing, N., Kimura, M., Bjørnstad, Å. and Klemsdal, S. S. 2008. Multilocus genotyping and molecular phylogentics resolve a novel head blight pathogen within the Fusarium graminearum species complex from Ethiopia. Fungal Genet. Biol. 45:1514-1522.

Proctor, R. H., Hohn, T. M. and McCormick, S. P. 1995. Reduced virulence of Gibberella zeae caused by disruption of a trichothecene toxin biosynthetic gene. Mol. Plant-Microbe Interact. 8:593-601.

Qu, B., Li, H. P., Zhang, J. B., Xu, Y. B., Huang, T., Wu, A. B., Zhao, C. S., Carter, J., Nicholson, P. and Liao, Y. C. 2008. Geographic distribution and genetic diversity of Fusarium graminearum and $F$. asiaticum on wheat spikes throughout China. Plant Pathol. 57:15-24.

Scoz, L. B., Astolfi, P., Reartes, D. S., Schmale III, D. G., Moraes,
M. G. and Del Ponte, E. M. 2009. Trichothecene mycotoxin genotypes of Fusarium graminearum sensu stricto and Fusarium meridionale in wheat from southern Brazil. Plant Pathology 58:344-351.

Suga, H., Karugia, G. W., Ward, T., Gale, L. R., Tomimura, K., Nakajima, T., Miyasaka, A., Koizumi, S., Kageyama, K. and Hyakumachi, M. 2008. Molecular characterization of the Fusarium graminearum species complex in Japan. Phytopathology 98:159-166.

Starkey, D. E., Ward, T. J., Aoki, T., Gale, L. R., Kistler, H. C., Geiser, D. M., Suga, H., Tóth, B., Varga, J. and O’Donnell, K. 2007. Global molecular surveillance reveals novel Fusarium head blight species and trichothecene toxin diversity. Fungal Genet. Biol. 44:1191-1204.

Tamura, K., Dudley, J., Nei, M. and Kumar, S. 2007. MEGA4: Molecular Evolutionary Genetics Analysis (MEGA) software version 4.0. Mol. Biol. Evol. 24:1596-1599.

Thompson, J. D., Higgins, D. G., and Gibson, T. J. 1994. CLUSTAL W: improving the sensitivity of progressive multiple sequence alignment through sequence weighting, positionspecific gap penalties and weight matrix choice. Nucleic Acids Res. 22:4673-4680.

Ueda, A., Nishimoto, H., Kato, N., Hirano, T. and Fukaya, M. 2007. Lineages and trichothecene mycotoxin types of fusarium head blight pathogens of wheat and barley in Todai district. Res. Bull. Aichi. Agric. Res. Ctr. 39:17-23.

Ward, T. J., Bielawski, J. P., Kistler, H. C., Sullivan, E. and O'Donnell, K. 2002. Ancestral polymorphism and adaptive evolution in the trichothecene mycotoxin gene cluster of phytopathogenic Fusarium. Proc. Natl. Acad. Sci. USA 99:92789283.

Ward, T. J., Clear, R. M., Rooney, A. P., O’Donnell, K. Gaba, D., Patick, S., Starkey, D. E., Gilbert, J., Geiser, D. and Nowicki, T. W. 2008. An adaptive evolutionary shift in Fusarium head blight pathogen populations is driving the rapid spread of more toxigenic Fusarium graminearum in North America. Fungal Genet. Biol. 45:473-484.

Yang, L., Lee, T., Yang, X., Yu, D. and Waalwijk, C. 2008. Fusarium population on Chinese barley show a dramatic gradient in mycotoxin profiles. Phytopathology 98:719-727.

Yli-Mattila, T., Gagkaeva, T., Ward, T. J., Aoki, T., Kistler, H. C. and O'Donnell, K. 2009. A novel Asian clade within Fusarium graminearum species complex includes a newly discovered cereal head blight pathogen from the Russian Far East. Mycologia 101:841-852.

Zhang, Z., Zhang, H., Lee, T., Chen, W. Q., Arens, P., Xu, J., Xu, J. S., Yang, L. J., Yu, D. Z., Waalwijk, C. and Feng, J. 2010. Geographic substructure of Fusarium asiaticum isolates collected from barly in China. Eur. J. Plant Pathol. 127:239-248. 